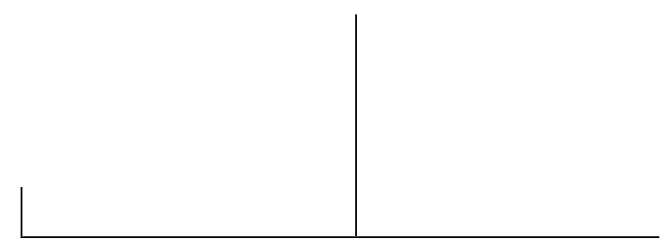

Rev. Latinoam. Psicopat. Fund., São Paulo, v. 12, n. 2, p. 356-365, junho 2009

\title{
Estigma e esquizofrenia: repercussões do estudo sobre discriminação experimentada e antecipada
}

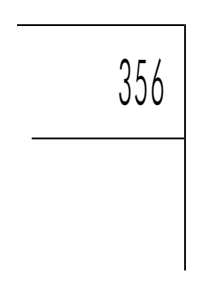

\begin{abstract}
Mônica Teixeira
O artigo "Padrão global da discriminação experimentada e antecipada contra pessoas com esquizofrenia: estudo transversal", publicado originalmente na Lancet, despertou interesse entre psiquiatras de vários países da Europa. As cartas enviadas por eles à revista inglesa foram reunidas aqui.
\end{abstract}

Palavras-chave: Discriminação, esquizofrenia, preconceito 
Na edição passada desta Revista, e em continuidade à colaboração com a revista Lancet, Observando a Medicina traduziu e apresentou a seus leitores o artigo "Padrão global de discriminação experimentada e antecipada contra pessoas com esquizofrenia: estudo transversal", de Graham Thornicroft e colegas. $\mathrm{O}$ artigo relata os resultados encontrados em pesquisa mundial na qual se pediu a pessoas com diagnóstico de esquizofrenia que avaliassem suas experiências de discriminação. $\mathrm{O}$ trabalho dos pesquisadores ingleses suscitou cartas à Lancet, publicadas na edição de 18 de abril do periódico inglês, e que podem ser lidas a seguir.

\section{Estigma e esquizofrenia}

Graham Thornicroft e colegas ${ }^{1}$ abordam um assunto relevante de saúde. Esquizofrenia é altamente prevalente ${ }^{2} \mathrm{e}$ as consequências da estigmatização são frequentemente devastadoras para os envolvidos. Embora o estudo em ques-

1. Thornicroft, G.; Brohan, E.; Rose, D.; Satorius, N.; Leese, M. for the INDIGO Study Group. Global pattern of experienced and anticipated discrimination against people with schizophrenia: a cross-sectional survey. Lancet, n. 373, p. 408-415, 2009.

2. Cardno, A.G.; Murray, R.M. The "classical" genetic epidemiology of schizophrenia. In: Murray, R.M.; Jones, R.B.; Susser, E.; van Os, J.; Cannon, M. (Eds.). The epidemiology of schizophrenia Cambridge: Cambridge University Press, 2003. p. 195-219. 


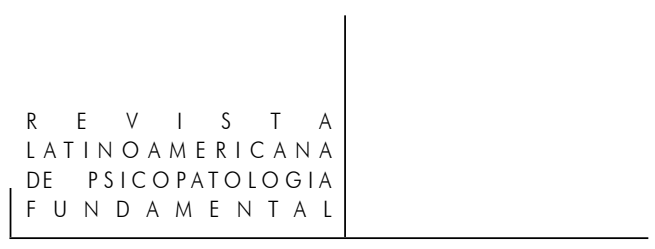

tão seja o primeiro a avaliar sistematicamente as experiências de pessoas com esquizofrenia em escala global, preocupa-me a interpretação dos resultados pelo fato de o artigo não abordar dois assuntos importantes.

Primeiro, uma das hipóteses mais amplamente testadas na pesquisa sobre esquizofrenia considera o papel causal da carga genética. Os estudos sobre a taxa de concordância para esquizofrenia, com gêmeos monozigóticos e heterozigóti$\cos ;{ }^{3}$ e a conexão observada entre a proximidade com um parente afetado na árvore familiar, oferecem fundamento para essas hipóteses. ${ }^{4}$ Thornicroft e colegas descobriram que mais de um terço dos participantes do estudo esperavam a discriminação, mesmo quando nenhuma discriminação houvesse sido experimentada antes. No entanto, o estudo não esclarece se os participantes tinham ou não história de esquizofrenia na família. Poderia esse resultado - da antecipação da discriminação - ser explicado pela experiência do participante a respeito do tratamento das gerações anteriores? Entrevistas e processo de coleta de dados mais abrangentes revelariam mais sobre as razões por trás dessas expectativas.

Outro resultado altamente documentado é a correlação inversa entre esquizofrenia e status socioeconômico. Várias medidas do status socioeconômico indicam risco maior entre camadas empobrecidas. ${ }^{5}$ Thornicroft e colegas não avaliaram o status socioeconômico, nem idade, sexo ou origem étnica, fatores que podem ativar as fontes de discriminação contra determinados indivíduos.

Mais estudos que levem esses temas em consideração poderiam oferecer maior direcionamento aos serviços de saúde mental para promover a inclusão social.

(Declaro não ter conflitos de interesse)

Bayanne Olabi

S0680946@sms.ed.ac.uk

Universidade de Edimburgo.

Colégio de Medicina e Veterinária, Edimburgo, EH8 9HP, UK.

3. Franzek, E.; Beckmann, H. Different genetic background of schizophrenia spectrum psychosis: a twin study. Am J Psychiatry, n. 155, p. 76-83, 1998.

4. Cardno, A.G.; Murray, R.M. The "classical" genetic epidemiology of schizophrenia. In: Murray, R.M.; Jones, R.B.; Susser, E.; van Os, J.; Cannon, M. (Eds.). The epidemiology of schizophrenia Cambridge: Cambridge University Press, 2003. p.195-219.

5. Gallagher, B.J.; Jones, B.J.; McFalls, J.A.; Pisa, A.M. Social class and type of schizophrenia. Eur Psychiatry, n. 21, p. 233-237, 2006.

Rev. Latinoam. Psicopat. Fund., São Paulo, v. 12, n. 2, p. 356-365, junho 2009 
Graham Thornicroft e colegas ${ }^{1}$ descobriram que a discriminação antecipada e a experimentada são consistentemente altas em países que registram doenças mentais. Embora o artigo seja detalhado e abrangente, alguns pontos devem ser esclarecidos.

Primeiro, é importante especificar o nível de educação dos participantes. ${ }^{2}$ Os dados foram coletados via entrevista e apoiados na avaliação subjetiva dos participantes sobre o preconceito. Quem tem nível mais alto de educação pode ser mais capaz de tolerar certas experiências do que aqueles com um nível mais baixo, e podem não julgar "estigmatizante" uma determinada experiência que alguém menos educado avaliaria assim. Além disso, participantes menos educados poderiam ter mais dificuldade para encontrar emprego, o que poderia aumentar a discriminação.

Em segundo lugar, o ambiente cultural deveria ser enfatizado. É difícil comparar o pobre ambiente rural com melhores condições urbanas. Adicionalmente, culturas diferentes têm atitudes diferentes em relação à saúde mental.

Em terceiro lugar, Thornicroft e colegas consideraram a severidade da doença dos participantes? É crucial estimar a discriminação negativa registrada frente à severidade da doença.

Finalmente, é importante comparar o tempo desde o diagnóstico da doença mental. Aqueles diagnosticados há muito tempo mais provavelmente enfrentaram discriminação do que os diagnosticados recentemente.

(Declaramos não ter conflitos de interesse)

Suning Chen, Wei Zhang, *Jian Zhang

biozhangj@yahooo.com.cn

Departamento de Farmácia, Xijing Hospital, Xi' an, Shaanxi, China (SC, JZ); Department of Biochemistry and Molecular Biology e State Key Laboratory of Cancer Biology, Fourth Military Medical University, Xi' an, Shaanxi, China (JZ, WZ)

1. Thornicroft, G.; Brohan, E.; Rose, D.; Satorius, N.; Leese, M. for the INDIGO Study Group. Global pattern of experienced and anticipated discrimination against people with schizophrenia: a cross-sectional survey. Lancet, n. 373, p. 408-415, 2009.

2. Norman, R.M.; Sorrentino, R.; Windell, D.; Manchanda, R. Are personal values of importance in the stigmatization of people with mental illness? Can J Psychiatry, n. 53, p. 848-856, 2008. 


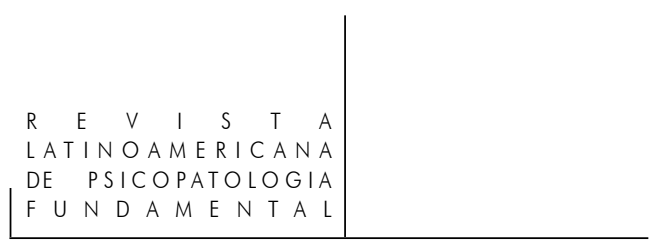

Em resposta ao artigo de Graham Thornicroft e colegas $^{1}$ e o Comentário de Beate Schulze, ${ }^{2}$ gostaríamos de compartilhar nossa experiência de luta contra o estigma da esquizofrenia.

$\mathrm{O}$ advento do tratamento comunitário da doença mental na Grécia, no início dos anos 1980, enfatizou o conceito holístico da doença mental, incorporando modelos psicossociais e biológicos, e levou ao desenvolvimento de um programa de reabilitação psicossocial igualmente concentrado em intervenções clínicas e psicoterapêuticas. ${ }^{3}$ Avaliações funcionais e clínicas regulares capacitaram os pacientes a participar do desenvolvimento de seus próprios planos terapêuticos individualizados.

Um resultado dessa participação foi a fundação, em 1984, da Associação contra o Preconceito de Doenças Mentais, ou Anagennisi ("renascimento", em grego). Desde então, a Anagennisi, com seus 385 pacientes membros, participa em uma série de programas de reabilitação vocacional na Europa e apoia ativamente projetos comunitários contra o estigma público com a promoção de uma rede de associações similares em toda a Grécia.

Recentemente, a Anagennisi foi aceita na Confederação Nacional Grega para Pessoas Deficientes e um paciente-membro foi eleito pela maioria para o conselho executivo, o que foi considerado um grande sucesso para pacientes com esquizofrenia e para pessoas com deficiências que abraçaram os membros da Anagennisi como seus iguais.

A identificação desses pacientes-membros com outras pessoas deficientes fortaleceram o esforço deles contra estigma público e estigma percebido. ${ }^{4}$ Talvez os próprios pacientes pudessem conduzir uma mudança na maneira condescendente com que a sociedade olha para eles.

(Declaramos não ter conflitos de interesse)

M. V. Karidi; C. Theleritis; N. C. Stefanis

vkarid@epipsi.gr

Psychosocial and Vocational Rehabilitation Unit, Greek University Mental Health Research Institute (UHMRI), Soranou Efesiou 2, Papayou 15601, Athens, Greece

1. Thornicroft, G.; Brohan, E.; Rose, D.; Satorius, N.; Leese, M. for the INDIGO Study Group. Global pattern of experienced and anticipated discrimination against people with schizophrenia: a cross-sectional survey. Lancet, n. 373, p. 408-415, 2009.

2. Schulze, B. Mental-health stigma: expanding the focus, joining forces. Lancet, n. 373, p: 362-363, 2009.

3. Karidi, M.V.; Papakonstantinou, K.; Stefanis, N. et al. Occupational abilities and performance scale: reliability-validity assessment factor analysis. Soc Psychiatry Psychiatr Epidemiol, n. 40, p. 417-424, 2005.

4. Watson, A.C.; Corrigan, P.; Larson, J.E.; Sells, M. Self-stigma in people with mental illness. Schizophr Bull, n. 33, p. 1312-1318, 2007. 
O artigo de Graham Thorbnicroft e colegas ${ }^{1}$ e o Comentário de Beate Schulze $^{2}$ são bem-vindas exceções a uma visão predominante na clínica psiquiátrica de que o estigma não é uma influência ambiental modificável capaz de afetar o curso e o prognóstico da psicose. ${ }^{3,4}$

Thornicroft e colegas argumentam a favor do desenvolvimento de intervenções clínicas. Estas, no entanto, permanecem subestudadas e subdesenvolvidas. Uma pista interessante sobre quais tratamentos podem funcionar é fornecida pela alta prevalência da discriminação antecipada. ${ }^{5}$ Esta descoberta sugere que não apenas a experiência do estigma em si, mas também a interação dinâmica com a pessoa, em termos de esquematização sobre si mesmo e sobre os outros, têm papel importante.

Há cerca de um ano, refletindo sobre modos de diminuir (a experiência de) estigmatização, pensei que uma intervenção centrada nas capacidades de indivíduos com severa doença mental de lidarem com situações, oferecida em um contexto empoderado de autoajuda e complementada por ajuda profissional, pode se constituir em uma intervenção adequada. Essas habilidades podem ser aprendidas com psicoeducação que se concentre em desenvolver respostas cognitivas não frustrantes e estratégias comportamentais alternativas, evitando o aparecimento de autoestigma e evitação.

O contexto da autoajuda é necessário como contraposição para a visão pessimista pervasiva sobre a esquizofrenia. Por exemplo, uma revisão destacada sobre esquizofrenia ${ }^{6}$ afirma o seguinte: "Uma vez que os sintomas da esquizofrenia ocorram (usualmente em adultos jovens), eles persistem por todo o tempo de vida

1. Thornicroft, G.; Brohan, E.; Rose, D.; Satorius, N.; Leese, M. for the INDIGO Study Group. Global pattern of experienced and anticipated discrimination against people with schizophrenia: a cross-sectional survey. Lancet, n. 373, p. 408-415, 2009.

2. Schulze, B. Mental-health stigma: expanding the focus, joining forces. Lancet, n. 373, p. 362-363, 2009.

3. van Zelst, C. Stigmatization as an environmental risk in schizophrenia: a user perspective. Schizophr Bull, n. 35, p. 293-296, 2009.

4. van Zelst $\mathrm{C}$. Which environments for $\mathrm{G} \times \mathrm{E}$ ? A user perspective on the roles of trauma and structural discrimination in the onset and course of schizophrenia. Schizophr Bull, n. 34, p. 1106-1110, 2008.

5. Thornicroft, G.; Brohan, E.; Rose, D.; Satorius, N.; Leese, M. for the INDIGO Study Group. Global pattern of experienced and anticipated discrimination against people with schizophrenia: a cross-sectional survey. Lancet, n. 373, p. 408-415, 2009.

6. Sawa, A; Snyder, S.H. Schizophrenia: diverse approaches to a complex disease. Science, n. 296, p. 692-695, 2002.

Rev. Latinoam. Psicopat. Fund., São Paulo, v. 12, n. 2, p. 356-365, junho 2009 
do paciente e são quase completamente incapacitantes". Perspectivas pessimistas como esta não conduzem ao desenvolvimento de habilidades pessoais para superar o estigma.

(Agradeço a $\mathbf{J}$ van Os por comentários a uma versão anterior desta carta. Declaro não ter conflitos de interesse)

\section{Catherine van Zelst}

c.vanzelst@sp.unimaas.nl

Department of Psychiatry, South Limburg Mental

Health Research and Teaching Network, EURON,

Maastricht University Medical Centre, PO BOX 616,

6200 MD Maastricht, Netherlands

\section{Resposta dos autores}

Bayanne Olabi levanta um ponto interessante sobre o papel da família na discriminação antecipada, particularmente em relação à discriminação antecipada ser ou não maior em indivíduos que testemunharam a discriminação de parentes. Não perguntamos aos participantes se eles tiveram uma história familiar de doença mental. A entrevista DISC (Discrimination and Stigma Scale) é estruturada como um instrumento que pede aos participantes o fornecimento de exemplos de discriminação experimentada ou antecipada em cada domínio da vida. A análise qualitativa desses dados, que serão relatados por nós no futuro, oferece recursos ricos para considerar quais fatores podem estar relacionados à antecipação da discriminação. No entanto, em próximos estudos, consideraremos a possibilidade de incluir itens que detalhem a história familiar da doença mental.

É também importante considerar que os membros da família podem eles mesmos antecipar ou experimentar o estigma (ou seja, estigma por associação) ou ainda ter preocupações sobre o estigma ou discriminação que o membro de sua família possa vir a sofrer (ou seja, estigma vicário). ${ }^{1}$ Esses cenários iluminam o papel complexo da família no processo de estigma. Examinaremos o pa-

1. Larson, J.E.; Corrigan, P. The stigma of families with mental illness. Acad Psychiatry, n. 32, p. 87-91, 2008.

Rev. Latinoam. Psicopat. Fund., São Paulo, v. 12, n. 2, p. 356-365, junho 2009 
pel da família na busca de ajuda como parte do Programa SAPPHIRE. Em relação a explicações genéticas para a doença mental, Phelan e colegas ${ }^{2}$ demonstraram que isto pode ter implicações positivas e negativas.

Relatamos sim dados sobre idade e sexo em nosso artigo, mas, devido à natureza colaborativa do trabalho, com participantes de 27 países, não coletamos informação sobre etnicidade. No entanto, reconhecemos sua importância e estamos realizando pesquisa sobre o assunto por meio do Programa SAPPHIRE. Nesse trabalho, examinaremos em detalhe experiências de discriminação múltipla por usuários de serviços de saúde mental.

Em resposta a Sunning Chen e colegas, examinamos sim o número de anos de educação em nosso estudo, a média da nossa amostra sendo 12,6 anos (DP 3.3). Nossas entrevistas, em sua maioria, foram feitas em centros urbanos, e a cada local foi pedido o recrutamento de entrevistados com diagnóstico de esquizofrenia que fossem representativos da área. Não coletamos outras informações sobre a severidade da doença.

Nosso modelo de regressão relatado apoia o comentário de que aqueles que foram diagnosticados há muito tempo devem registrar um maior número de experiências de discriminação do que aqueles diagnosticados recentemente. De fato, observamos que o tempo desde o diagnóstico esteve associado com experiências de discriminação crescentemente negativas: assim, quando o tempo desde o diagnóstico foi de "menos de cinco anos" para "maior que quinze anos", a discriminação negativa aumentou de 1,9 unidade em média.

Os comentários de M. V. Karidi e colegas e de Catherine van Zelst são um bem-vindo meio de dividir experiências e continuar a discussão sobre possíveis intervenções para reduzir o estigma e a discriminação. ${ }^{3}$

Declaramos não ter conflitos de interesse

Graham Thornicroft, Elaine Brohan, Diana Rose, Norman Sartorius, Morven Leese g.thornicroft@iop.kcl.ac.uk

Health Service and Population Research Department, Institute of Psychiatry, King's College London SE5 8AF, UK (GT, EB, DR, ML); and 14 Chemin Colladon, Geneva, Switzerland (NS)

2. Phelan, J.C.; Yang, L.H.; Cruz-Rojas, R. Effects of attributing serious mental illnesses to genetic causes on orientations to treatment. Psychiatr Serv, n. 57, p. 382-387, 2006.

3. Schulze, B. Mental-health stigma: expanding the focus, joining forces. Lancet, n. 373, p. 362$363,2009$.

Rev. Latinoam. Psicopat. Fund., São Paulo, v. 12, n. 2, p. 356-365, junho 2009 


\section{Resumos}

(Estigma y esquizofrenia: repercusiones del estudio sobre discriminación experimentada y anticipada)

El artículo "Norma global de la discriminación experimentada y anticipada contra personas con esquizofrenia: estudio transversal", publicado originalmente en Lancet, despertó interés entre psiquiatras de varios países de Europa. Las cartas enviadas por ellos a la revista inglesa fueron reunidas aquí.

Palabras claves: Discriminación, esquizofrenia, prejuicio

(Stigmatisation et schizophrénie: répercussions de l'étude sur la discrimination vécue et anticipée)

L'article "Profil mondial de la discrimination vécue et anticipée contre les personnes atteintes de schizophrénie: une enquête transversale", initialement publié dans le Lancet, a suscité l'intérêt des psychiatres dans plusieurs pays d'Europe. Leurs lettres adressées à la revue anglaise se trouvent ici réunies.

Mots clés: Discrimination, schizophrénie, préjugé

(Stigma and schizophrenia: repercussions on the study about experienced and expected discrimination )

The article "Global standard of experimented and expected discrimination against persons with schizophrenia: transversal study," originally published in British journal Lancet, stirred up considerable interest among psychiatrists in various European countries. Letters many of them sent to the journal are brought together here.

Key words: Discrimination, schizophrenia, prejudice

Citação/Citation: TeIXeIra, M. Estigma e esquizofrenia: repercussões do estudo sobre discriminação experimentada e antecipada. Revista Latinoamericana de Psicopatologia Fundamental, São Paulo, v. 12, n. 2, p. 356-365, jun. 2009.

Editor do artigo/Editor: Mônica Teixeira.

Recebido/Received: 10.5.2009/ 5.10.2009 Aceito/Accepted: 13.5.2009/ 5.13.2009

Copyright: () 2009 Associação Universitária de Pesquisa em Psicopatologia Fundamental/ University Association for Research in Fundamental Psychopathology. Este é um artigo de livre acesso, que permite uso irrestrito, distribuição e reprodução em qualquer meio, desde que o autor e a fonte sejam citados/This is an open-access article, which permits unrestricted 
use, distribution, and reproduction in any medium, provided the original author and source are credited.

Financiamento/Funding: $\mathrm{O}$ autor declara não ter sido financiado ou apoiado/The author has no support or funding to report.

Conflito de interesses: $\mathrm{O}$ autor declara que não há conflito de interesses/The author declares that has no conflict of interest.

\section{Mônica TeixeIra}

Jornalista; psicanalista; coordenadora geral do programa Universidade Virtual do Estado de São Paulo na TV Cultura (São Paulo, SP, Brasil); diretora de redação do boletim eletrônico Inovação Unicamp (Campinas, SP, Brasil).

Av. Higienópolis, 318/13

01238-001 São Paulo, SP, Brasil

e-mail: armonica@uol.com.br 